\title{
Content Based Image Retrieval with Fuzzy Geometrical Features
}

\author{
Minakshi Banerjee and Malay K. Kundu \\ Machine Intelligence Unit, Indian Statistical Institute \\ 203, B. T. Road, Kolkata - 700 108, INDIA \\ e-mail: \{minakshi_r, malay\}@isical.ac.in
}

\begin{abstract}
This paper presents a robust technique for Content Based Image Retrieval (CBIR) using fuzzy edge map of an image. Fuzzy compactness vector is computed from fuzzy edge map thresholded at different levels of the unsegmented image, which also incorporates gray level contrast information embedded in the edges. The resemblance of two images is defined as the similarity between the computed feature vectors.
\end{abstract}

\section{INTRODUCTION}

Due to advances in internet and image databases, Content Based Image Retrieval (CBIR) has become a challenging research area. In CBIR paradigm [1] images are automatically indexed using visual contents of an image which are mainly color, texture and shape. These visual features are useful in characterizing an image [2] although they cannot capture any semantic information of an image. Recent trend in CBIR research is to develop efficient features for simpler characterization of images and related matching techniques so that it can handle real life images. Fuzzy set theoretic approach may be found suitable in CBIR applications because the users are interested in results according to similarity (closeness) rather than equality (exactness). Due to the inherent advantages of this approach of handling uncertainties, Fuzzy set theoretic model has become appropriate for applications where there is a possibility of incompleteness or perturbation of data. Chen and Wang used fuzzy logic approach for region based image retrieval. In their approach an image is represented by a set of segmented regions [3], each of which is represented by a set of fuzzy features ( color, texture, shape ). Similarity between two images is defined as the similarity between the fuzzy features and quantified by the similarity measure. In our proposed technique no prior segmentation is required. Our technique is mainly suitable for the class of images having single object in uniform or weak textured background but can also be used with certain degree of accuracy for other type of images. Unlike other CBIR techniques [3], in the present technique no additional cost of pre segmentation is involved. Edge is an important and strong visual feature as it represents the majority of visual information with a limited number of pixels. Region boundaries are characterized by strong edges and approximately represents the shape of the different regions. For this reason, edges can be used as an important feature for CBIR applications. It is also to be noted that single feature may not be sufficient to generate expected results for different images. Other features like color, texture etc. are also used along with edge feature to achieve higher accuracy. Zhou and Huang [4] proposed structural features extracted from edge maps for CBIR. Histogram of blurred images are also used for defining shape features because histograms of blurred images are strongly influenced by the original shapes of different regions in it [5]. Using recent edge detection method, Wang, Gray and Wiederhold [6] presented a technique in which edges of the images can be extracted by wavelet transform. The edge image are then classified based on the invariant moments. Achard, Devars and Lacassagne proposed a shape based feature combining gray level information [7] by thresholding the image at different gray levels. Compactness of the object is computed over each gray level for shape comparison. This method is computationally very expensive and cost is proportional to the number of gray levels considered. Regarding feature selection one should keep in mind that the features should not be computationally expensive, should be noise tolerant and invariant to rotation, translation and scaling. Considering the following properties the major contributions are as follows.

We have used the blurred image as input and used the concept of Top and Bottom of the intensity surface to extract possible candidates for the edge map. This map is converted to fuzzy gradient map by measuring the relative intensity difference over small neighborhood. The pixels are categorized as Weak, Medium, Strong based on their contrast membership value. Multilevel thresholding above certain membership value is performed to find multilevel fuzzy edge map, from which (fuzzy compactness vector) is computed. This geometrical feature is invariant to Rotation Translation and Scaling by definition and physically means the maximum area that can be encircled by the perimeter. The similarity between the feature vectors of two images are computed by the widely used standard Euclidean distance metric.

In the next section we briefly describe the mathematical model used in this work. The feature extraction methodology and the algorithm is described in section III. In section IV we present critical remarks about the performance and finally conclude our study in section $\mathrm{V}$.

\section{FUZZY IMAGE MODEL}

Feature vectors for characterizing an image are computed using fuzzy set theoretic approach. Gray level images are inherently fuzzy in nature due to the uncertainty that exists 
in locating the exact position of the boundary which separates the object from background. Gaussian smoothing is important to clean the image. Blurring of edges gives rise to positional uncertainty which demands Fuzzy set theoretic approach to handle such uncertainties.

\section{A. Fuzzy set representation of an image}

An image $\mathrm{X}$ of $M \times N$ size and $\mathrm{L}$ levels can be considered as an fuzzy subset $A^{\prime}[8]$ in a space of points $\mathrm{X}=\{x\}$. Each point in $\mathrm{X}$ can be characterized by a membership function $\mu_{A}\left(x_{m n}\right) \cdot A^{\prime}=\left\{\left(\mu_{A}\left(x_{m n}\right), x_{m n}\right\}\right.$ $m=1,2, . . M, n=1,2, . . N$ where $0 \leq \mu_{A}\left(x_{m n}\right) \leq 1.0$.

Fuzzy Compactness : For a $M \times N, \mu_{m n}$ array the fuzzy compactness $\operatorname{comp}(\mu)$, [9] is defined as follows .

$$
\operatorname{comp}(\mu)=\frac{a(\mu)}{p^{2} \mu}
$$

where $a(\mu)$ and $p(\mu)$ are the fuzzy area and perimeter of $(\mu)$. Where $a(\mu)$ and $p(\mu)$ are given by (2) and (3).

$$
a(\mu)=\sum_{m} \sum_{n} \mu_{m n}
$$

$p(\mu)=\sum_{m=1}^{M} \sum_{n=1}^{N-1}\left|\mu_{m n}-\mu_{m, n+1}\right|+\sum_{n=1}^{N} \sum_{m=1}^{M-1}\left|\mu_{m n}-\mu_{m+1, n}\right|$

\section{B. Feature extraction for CBIR}

The border region between two uniform intensity surfaces are our region of interest. The pixels of these regions are the possible candidates for finding the fuzzy edge map and computation of features.

Plateau Top, Plateau Bottom Edges in an image are the boundary between two relatively distinct uniform intensity surfaces. The uniform intensity surfaces are defined in terms of Plateaus. Plateaus are defined as connected subsets of uniform intensity pixels. This definition can be taken as the special case of fuzzy plateaus introduced by Rosenfield [10].

Plateau : Let $S$ denote the set of all pixels in an image. By a plateau in $S$ is meant a maximum connected subset $S_{p}$ on which the intensity has a constant value. In other words $S_{p} \in S$ is a Plateau iff

(i) $S_{p}$ is connected. (ii) $I(P)=I(Q)$ for all P,Q $\in S_{p}$ (iii) $I(P) \neq I(Q)$ for all pair of neighboring points $P \in S_{p}$ and $Q \notin S_{p}$

Clearly $P \in S$ belongs to one Plateau.

We call the Plateau $S_{p}$ a Top if it's gray value is a local maximum i.e. $I(P) \geq I(Q)$ for all pairs of neighboring point $P \in S_{p}$ and $Q \notin S_{p}$. Similarly we call $S_{p}$ a Bottom if it's gray value is a local minimum. The gray values of all elements of Plateau Top is greater than the gray value of any element of the border and the gray values of any element of a Plateau Bottom is less than gray values of the border. Let $S_{p t}$ be a Plateau Top and $S_{p b}$ a Plateau Bottom. The pixels in border region $\mathrm{B}\left(S_{p t}, S_{p b}\right)$ between two Plateau Top and
Plateau Bottom can be defined as the points which are eight neighbors of at least one element of $S_{p t}, S_{p b}$. Considering a $3 \times 3$ neighborhood around a pixel Uma Shankar and Chaudhury proposed a method for characterizing extreme components of an image [11]. The pixels are labeled as pels of a (a) Plateau Top for which the pixel is greater or equal to maximum in it's $3 \times 3$ neighborhood (b) Plateau Bottom for which the pixel is less or equal to minimum in it's $3 \times 3$ neighborhood.(c) The remaining pels are Candidate pels for forming fuzzy gradient map.

\section{Membership function $\mu_{m}(P)$ for determining edge contrast}

We have used exponential form of membership function [12] as shown in (4). The motivation behind using this type of function is to select significant edge pixels from the slowly varying slope region near the cross over point $(0.5)$ membership value of the membership function. As the pixels of very low contrast membership value arises mainly due to imaging defects of the uniform surfaces, these pixels are to purged and the significant edge points are to be separated into different categories. The border region of the plateaus of the blurred (Gaussian) image is taken as input for finding the fuzzy gradient map and the total dynamic range of 0 to 256 is considered. The line joining two opposite pixels which makes four distinct lines are considered.

$$
\mu_{m}(P)=k_{m} \exp (-x)
$$

Considering Fig. 1, if $d_{a c}$ is the gray level difference between the opposite neighbors about candidate pixel in a direction say, $(0,4)$ and $d_{a l}$ is the gray level difference in the perpendicular direction say $(2,6)$ then $x=\frac{1+d_{a l}}{1+d_{a c}}$. We compute the magnitude membership values in four direction pairs. The maximum value out of the four directions is assigned to the candidate pixel. Edge direction is perpendicular to the direction of maximum difference value [13]. Let $I_{m n}$ be the gray level of the point $\mathrm{P}(\mathrm{m}, \mathrm{n})$. If $d_{a l}=\left|I_{m, n-1}-I_{m, n+1}\right|$ then $d_{a c}=\left|I_{m-1, n}-I_{m+1, n}\right|$ vice-versa as shown in Fig. 1 .

The value of $k_{m}$ can be estimated from the following condition. $\mu_{m}(P)$ is ranging from $[0,1]$. Minimum value of $d_{a l}=0$ and maximum value of $d_{a c}$ say $\mathrm{L}$ should map the membership value to into 1 . Different value of $\mathrm{L}$ should give rise to different value of $k_{m}$ hence different crossover point $\left(\mu_{m}(P)=0.5\right)$. Higher membership value characterizes strong edge pixels, and lower membership values will characterize weak edge pixels shown in Fig. 3 if they are already present in the image. The pixel contrast enhancement can be performed [12] by transforming the membership value as shown in (5) where $T_{1}(\mu(P))=T^{\prime}((\mu(P))$.

$$
\begin{aligned}
\mu_{m}(P) & =2 \times \mu_{m}(P)^{2} & & 0 \geq \mu_{m}(P) \leq 0.5 \\
& =1-2 \times\left(1-\mu_{m}(P)\right)^{2} & & 0.5 \geq \mu_{m}(P) \leq 1.0
\end{aligned}
$$




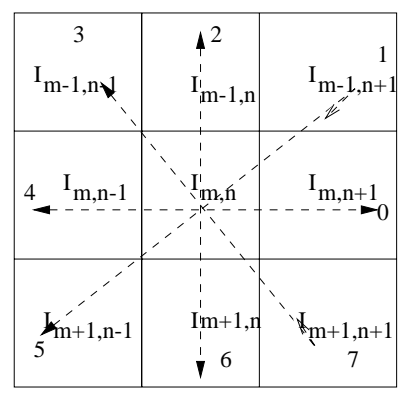

Fig. $1 . \quad 3 \times 3$ neighborhood of the candidate pixel.

\section{Membership function $\mu_{d}(P)$ for degree of linking}

We consider a $M \times N, \mu_{m}(P)$ array. In the neighborhood of a pixel $\mathrm{p}(\mathrm{m}, \mathrm{n})$ if there exits a pixel $p\left(m^{\prime}, n^{\prime}\right)$ such that,

$$
\left|\mu_{m n}-\mu_{m^{\prime} n^{\prime}}\right| \leq T
$$

where ( $\mathrm{T}$ is the non-negative threshold) are considered similar in magnitude. Again if

$$
\left|\operatorname{ang}(m, n)-\operatorname{ang}\left(m^{\prime}, n^{\prime}\right)\right| \leq A
$$

where $\mathrm{A}$ is the angle threshold are considered similar in direction [13]. The two above properties are combined to link similar pixels and the degree of linking is expressed by a new membership function as expressed in (8). The number of pixels possessing $\mu_{m}(P)$ above some threshold value $(\geq 0.5$ ) and $\leq 1.0$ and simultaneously having directions which didn't differ more than 45 degree in a neighborhood of $3 \times 3$ or $5 \times 5$ window size are linked. The membership function is of the form.

$$
\mu_{d}(P)=k_{d} \exp (-x)
$$

where $x=\frac{1}{n 1+n 2+n 3}, \mathrm{n} 1$ denotes is the number of edge pixels possessing same angle to that of the candidate pel, $\mathrm{n} 2$ is the number of edge pixels having +45 degree difference with the candidate pixel, $\mathrm{n} 3$ is the number of edge pixels having angle -45 degree difference with the candidate pixel. The value of $k_{d}$ is estimated by the maximum number of connected pixels say $\mathrm{K}$ along any of the four directions within the defined window. With $x=\frac{1}{K}$ maps the membership to 1 . Changing the value of $k_{d}$ will in effect change the number of connected pixels over a defined window.

\section{FEature eXtraction Methodology}

The intensity image matrix $I(m, n)$ is convolved with Gaussian function and is resulted into to a blurred image matrix $I_{b}(m, n)$.

$$
I_{b}(m, n)=I(m, n) * G(x, y)
$$

where $G(x, y)=\frac{1}{\sqrt{2} \pi \sigma} e^{-\frac{x^{2}+y^{2}}{2 \sigma^{2}}}$ where $\sigma$ effectively determines the window size of the filter which in turn determines the degree of smoothing. We are interested in finding the transition between the Plateau region consisting of the possible edge candidates $P_{c}$ which acts as an input for finding the fuzzy gradient map. The points of this region are characterized by $\mu_{m}(P)$ representing the edge strength based on the contrast value in the interval $[0,1]$ shown in equation below.

$$
s_{n}=\left\{\left(\mu_{m}(P), P_{c}\right)\right\}
$$

The gradient map $\mu_{m}(P)$ is thresholded at different levels using $(\alpha-c u t)[12]$ with membership values greater from 0.5 onwards. Let the candidates of the multilevel gradient map be represented by the equation given below.

$$
s_{n \alpha}=\left\{\left(P \in s_{n}: \mu_{m}(P) \geq \alpha\right\}\right.
$$

where $0.5 \geq \alpha \leq 1$.

Now $s_{n \alpha}$ is taken as input to find out the refined edge map using the notion of highest connectivity which resulted in a subset $s I_{n \alpha}$ as shown in (12). Points which are preserving highest connectivity within a defined window are extracted using the membership function $\mu_{d}(P)$ shown in (8).

$$
s \prime_{n \alpha}=\left\{\left(x \in s_{n \alpha}: \mu_{d}(P) \geq \alpha \prime\right\}\right.
$$

$\alpha^{\prime}$ is $\simeq 1$ to change the degree of connectivity between the adjacent pixels to the highest value. Fuzzy compactness feature $\operatorname{comp}(\mu)$ is computed on $s \prime_{n \alpha}$ using eqn.1.

\section{Algorithm}

Step1 : Gaussian filtering is performed on the image.

Step2 : Each pixel is labeled as (a) Plateau Top or maxima or (b) Plateau Bottom or minima or (c) Candidate pel

Step3 : If the labeled pixel is a candidate pel, it's contrast membership value $\mu_{m}(P)$ and it's edge direction is estimated. The points thresholded above different membership values $\left(\mu_{m}(x) \geq 0.5\right)$ are selected.

Step4 : Candidate pixels are nonlinearly intensified by contrast enhancement operation.

Step5 : Candidate pixels similar in edge magnitude and direction are linked to get a refined edge map.

Step6 : Fuzzy compactness feature vector is computed from the edge map.

Step7 : Euclidean distance is computed between the feature vector elements of the query image and images in the database and ranked according to distance.

$\left[\operatorname{comp}\left(\mu_{1}\right), \operatorname{comp}\left(\mu_{2}\right), \operatorname{comp}\left(\mu_{3}\right)\right]$ is computed from the refined edge map obtained at different threshold using (1).

\section{A. Choice of membership function and thresholds}

The choice of the exponential form of membership function can be supported considering the human psycho visual facts [14] as given by Wabers law. Considerable experimental evidence indicates that perceived brightness is a logarithmic 
function of the light incident on the eye [13]. The piece wise linear plot of $\log \frac{\delta I}{T}$ against $\log$ ( (where I denotes the intensity at a point and $\delta$ is the perceivable change in intensity) has a shape as shown in Fig.10. In psychology, contrast refers to the ratio of difference in luminance of intensity I at point and its surroundings. By choosing contrast membership an exponential function defined $\mu_{m}(P)$ by (4) the variation of $\log \mu_{m}(P)$ against $\mathrm{x}$ is made to support Waber's law. The edge map obtained using exponential and $\pi$ type membership function are shown in Figs.5(b) and 7. It is seen that a better edge map is obtained in by using exponential functions $\mu_{m}(P)$ and $\mu_{d}(P)$ in comparison to widely used $\pi$ type function [9]. In the later case the discrimination between the edge pixels of different contrast membership value is detoriated due to more visual nonlinearity which results in broken edge points. Also we have chosen a global thresholding scheme. Such global properties are important for overall characterization of an image. The images are thresholded above different membership values to give a multiscale value of the global property.

\section{B. Robustness of the features for application to CBIR.}

It is evident from the above mentioned feature extraction method, that we are able to classify the edge pixels based on their contrast membership value. The edge pixels are classified as weak, medium and strong edge pixels. Due to pre contrast enhancement operation as shown in (5) the strong edge pixels possessing contrast membership value greater then 0.5 are enhanced and widely separated and the weaker pixels those below 0.5 value are squeezed. By thresholding at higher $\alpha-c u t$ planes say (0.9) the pixels with higher contrast membership values are retained which grossly represents the stronger boundary pixels as shown in Fig. 5(c). At lower threshold, say at (0.7) membership value the edge map contains strong, medium, and weak type edge pixels as shown in Fig. 5(b). Similarly at threshold value $(0.8)$ the edge map contains medium and higher contrast pixels. The 3-D fuzzy compactness vector is computed and each component represents fuzzy compactness value obtained from the fuzzy edge map at different threshold. This vector is used for indexing images.

\section{EXPERIMENTAL RESULTS}

Our database consists of 120 gray level images $(256 \times$ 256 ) of different objects downloaded mainly from Google engine (www.google.com), Olivetti Face database(ftp : //ftp.uk.research.att.com : pub/data/att_faces.tar.Z) and some synthetically generated images. The images are mainly of single object both with textured and non textured background. The size of the gaussian smoothing filter is fixed to $3 \times 3$ pixels and value of $\sigma$ to 1.5 . The experiment is performed in ( SUN microsytems Ultra 60 ) system using MATLAB package.

The experimental results are shown from Figs. 2 to Figs. 9. Fig. 2(a) is a synthetically generated image and it's corresponding multilevel gradient map as obtained from (4) is

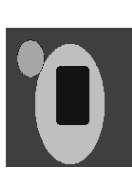

(a)

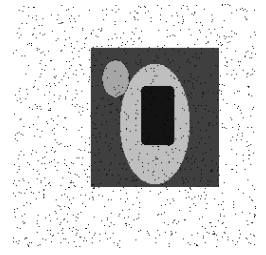

(b)
Fig. 2. (a) original image (b) noise introduced

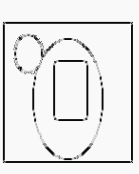

(a)

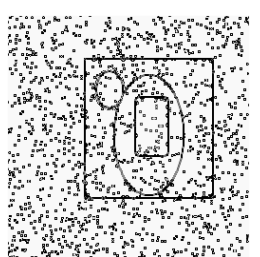

(b)
Fig. 3. Multilevel gradient map thresholded at $\mu_{m}(P) \geq$ (a) 0.6 (b) 0.6

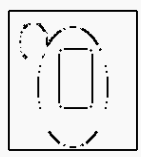

(a)

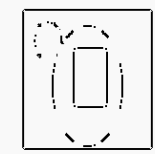

(b)

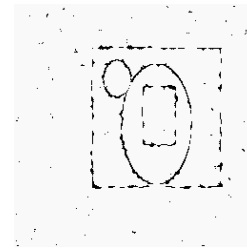

(c)
Fig. 4. Edge map obtained at $\mu_{m}(P) \geq$ (a) 0.7 (b) 0.8 (c) 0.6 (noisy) and $\mu_{d}(P) \simeq 1.0$. Candidates plotted as crisp edge point.

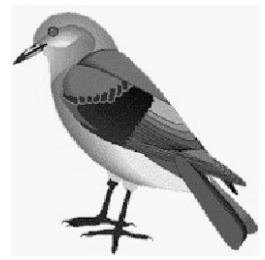

(a)

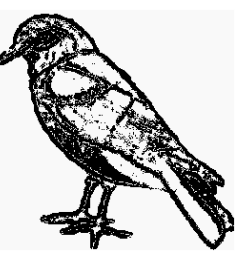

(b)

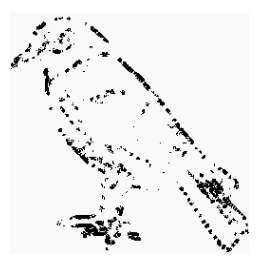

(c)
Fig. 5. (a) Original image (b) Edge map obtained at $\mu_{m}(P) \geq 0.7$ (c) 0.9 and $\mu_{d}(P) \simeq 1.0$. Candidates plotted as crisp edge point.

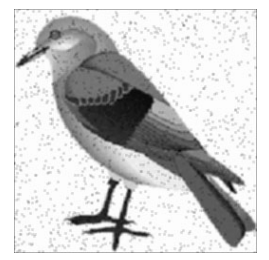

(a)

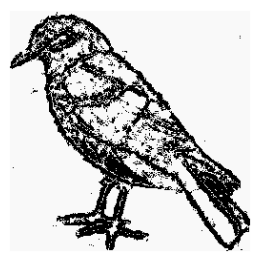

(b)

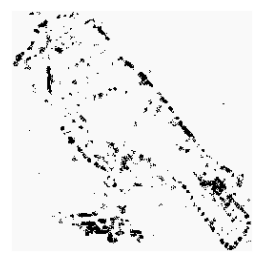

(c)
Fig. 6. (a) Original image (noisy background) (b) Edge map obtained at $\mu_{m}(P) \geq 0.7$ (c) 0.9 and $\mu_{d}(P) \simeq 1.0$. Candidates plotted as crisp edge point. 

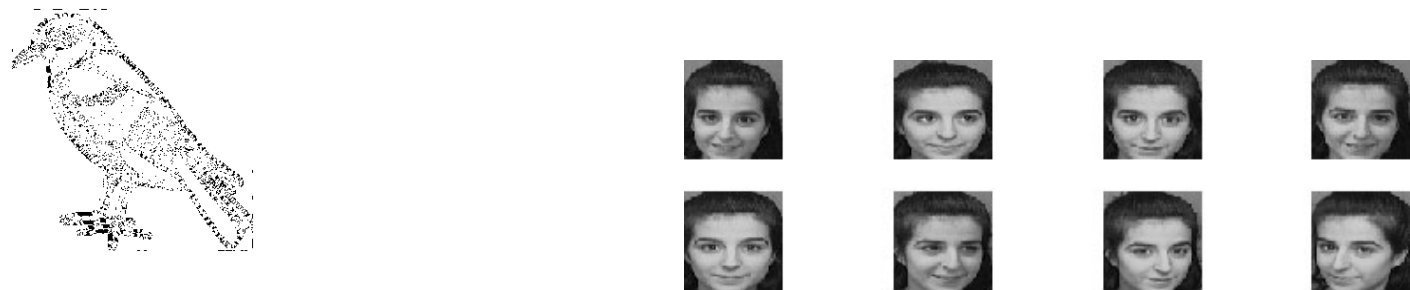

Fig. 7. Edge map obtained at $\mu_{m}(P) \geq 0.7$ with $\pi$ type membership function.

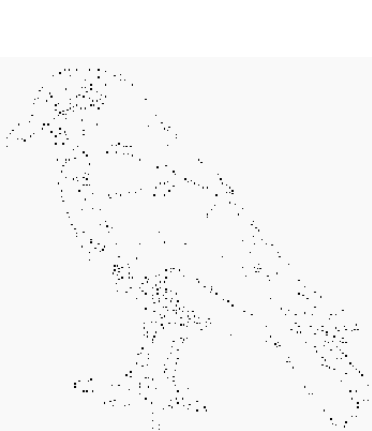

(a)

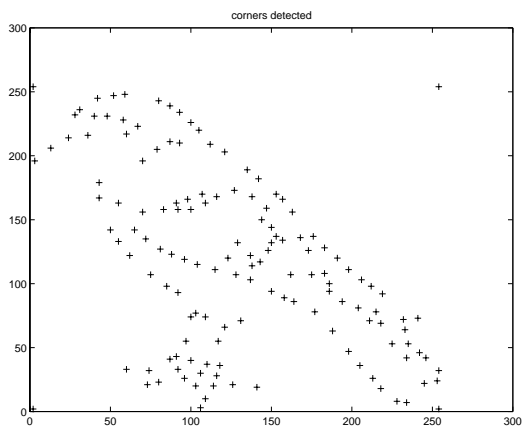

(b)
Fig. 8. (a) Some significant curvature points from the edge map. (b) Harris corner detector

shown in Fig. 3(a). The noisy image is shown in Fig. 2(b). The corresponding gradient map is shown in Fig. 3(b). Figs. 4(a),(b) shows the refined edge map of Fig. 2(a) at different threshold $\left(\mu_{m}(P) \geq 0.7,0.8\right)$. Fig. 4(c) is the refined edge map for the noisy image of Fig. 2(b). Considering Fig. 3(b) and Fig. 4(c) it is seen that the proposed technique has a good noise tolerance. Our aim is to select significant candidates from the multilevel edge map image containing meaningful shape and contrast information. From our proposed algorithm, it is observed from Fig. 3(a) that points of the curved regions are of lower membership value compared to the pixels belonging to the straight line. This property is used to extract points from curved regions of the edge map as shown in Fig. 8(a). There are different existing techniques for finding high curvature points. A multispectral corner detector has been proposed by [15]. However our algorithm is being modified to capture high curvature points precisely which may improve the retrieval score. The corner map of a standard corner detector proposed by Harris is shown in Fig. 8(b). It is possible to make a nonlinear intensification of the edge pixels depending upon the membership value as shown in (5) to categorize the edge pixels according to membership values as seen from Figs. 5 and 6. The edge map of the object in uniform and weak textured background is shown in Figs. 5 and Fig. 6. We select

TABLE I

PERFormance EVAluation \% .

\begin{tabular}{|c|c|c|c|c|}
\hline Image & $n_{1}$ & $n_{2}$ & Recall rate & Precision rate \\
\hline Fig.9(a) & 9 & 10 & 90 & 45 \\
\hline Fig.9(b) & 11 & 11 & 100 & 55 \\
\hline Fig.9(c) & 5 & 6 & 90 & 30 \\
\hline
\end{tabular}
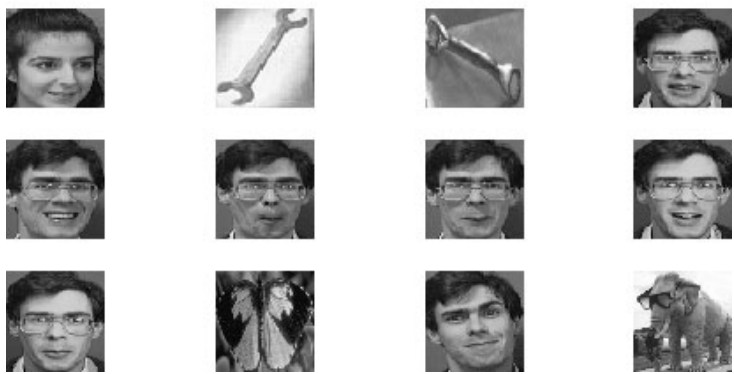

(a)
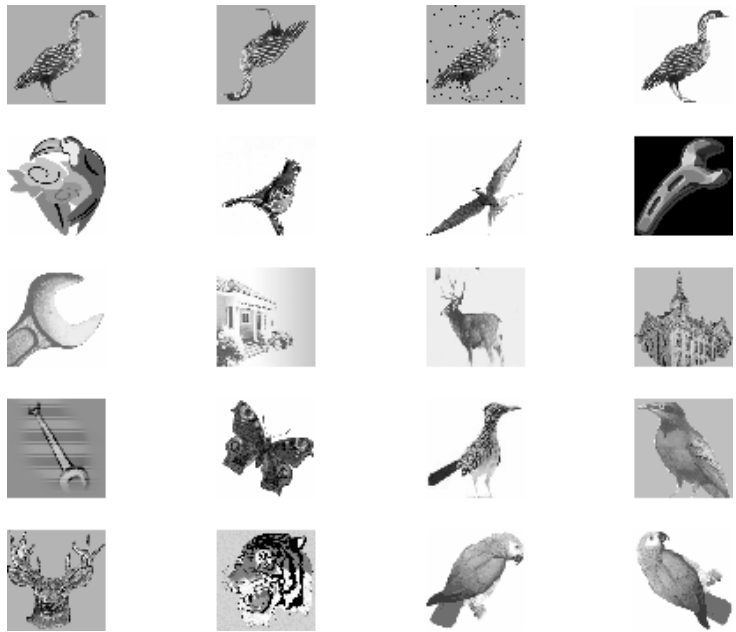

(b)
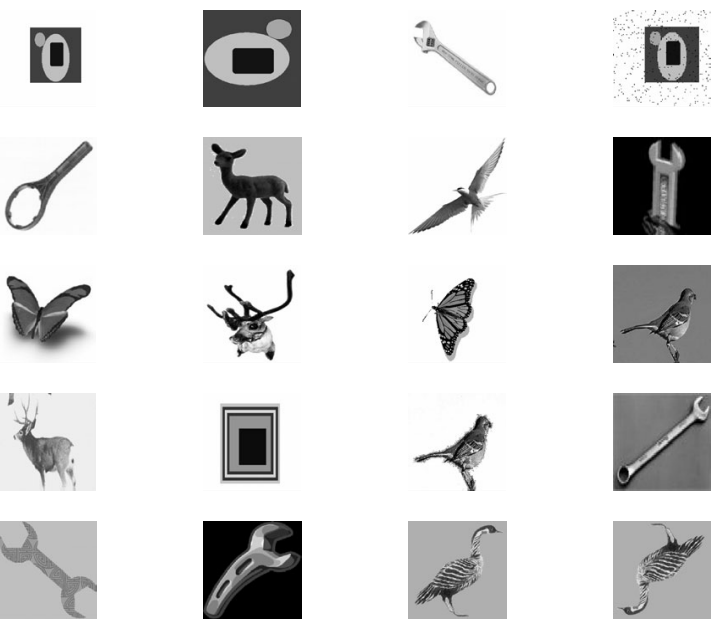

(c)

Fig. 9. Retrieved result, with top left image as the query image. 


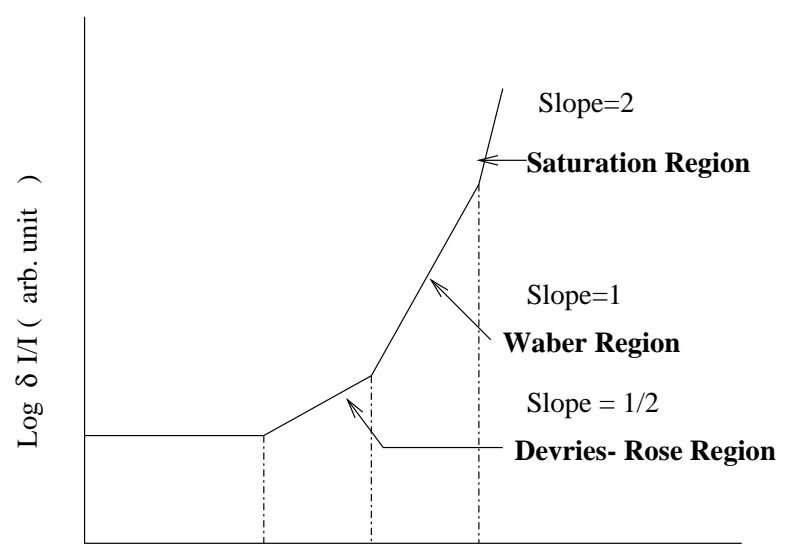

Log I (arb. unit)

Fig. 10. Plot of $\log \frac{\delta I}{I}$ against $\log I$ with linear approximation for different regions.

a particular image as query and based on the widely used Euclidean distance, computed between the feature vectors the images are ranked left to right and top to bottom according to the increasing order of distance and top twenty images are selected. These results are shown in Figs. 9(a), (b) and (c). The retrieval performance is tested for images having single objects (rotated, scaled, noisy condition) in uniform and weak textured background. The features are computed as follows: $\operatorname{comp}\left(\mu_{1}\right)$ and $\operatorname{comp}\left(\mu_{2}\right)$ are computed from the edge map thresholded at $\mu_{m}(P) \geq 0.8$ and 0.9. $\operatorname{comp}\left(\mu_{3}\right)$ is computed from significant points of the edge map shown in Fig. 8(a) obtained by thresholding at $\mu_{m}(P) \geq 0.7$ using (8). Although we have selected three thresholding levels of membership but the variation of selection of thresholding level will not change the result drastically. To evaluate the performance of retrieval we consider the standard retrieval benchmarks such as Recall rate , Precision rate [16]. Let $n_{1}$ be the number of images retrieved in top 20 positions that are close to the query. Let $n_{2}$ represent the number of images in the data base similar to the query. Evaluation standards Recall rate (R) is given by $\frac{n_{1}}{n_{2}} \times 100 \%$ and Precision rate $(\mathrm{P})$ is given by $\frac{n_{1}}{20} \times 100 \%$. It is also seen from the experimental results shown in Fig.9 that some dissimilar images attains higher rank and comes between the similar images. Such results can be explained considering the fact we have used a global measure (fuzzy compactness) for computing similarity. As the fuzzy compactness is computed from the edge map irrespective whether the pixel belongs to the object or background, the resultant compactness value can have contribution both from the object and background. In case when the background is textured the contribution from the background is more in comparison to the case where the background is uniform which results in a higher degree of error in computing the compactness of the object of interest.

\section{CONCLUSION}

As is evident from the experimental results fuzzy compactness provides an fairly good tool for indexing and can be able to retrieve images which are approximately similar in 2D shape (not occluded)from a heterogeneous database whose classes are not known apriori. The retrieved candidates can be further processed with other features for finer matching in the hierarchical search. Fuzzy compactness feature is computed from the edge map containing edge points only. Number of edge points are a small fraction of the total number of pixels in an image. It is an inexact method and it's performance is best achieved when queried with single objects and having non textured background. The method could be further improved if we use some other features like texture in association with the present work.

\section{ACKNOWLEDGMENT}

Minakshi Banerjee is grateful to the Council of Scientific and Industrial research, New Delhi, India, for providing her a research fellowship vide, grant (no.9/93(60)/99-EMR.I) to carry out her research work.

\section{REFERENCES}

[1] A. W. M. Smeulders, M. Worring, S. Santini, A. Gupta, and R. Jain, "Content-based image retrieval at the end of the early years.," IEEE Transactions on Pattern Analysis and Machine Intelligence, vol. 22(12), pp. 1349-1380, 2000.

[2] V. N. Gudivada and V. V Raghavan, "Content based image retrieval systems," IEEE.Comput., vol. 28(9), pp. 18-22, 1995.

[3] Y. Chen and J. Z.Wang, "A region-based fuzzy feature approach to content-based image retrieval.," IEEE Transactions on Pattern Analysis and Machine Intelligence, vol. 24(9), 2002.

[4] X. S. Zhou and T. S. Huang, "Edge-based structural features for contentbased image retrieval," Pattern Recognition Letters, vol. 22, pp. 457468, 2001.

[5] L. da Fontoura costa and R. Marcondes, Shape analysis and classification, CRC Press, USA, 2001.

[6] J.Z. wang, R.M. Gray, and G. Wiederhold, "System for screening objectionable images.," Computer Communications Journal, vol. 21(15), pp. 1355-1360, 1998.

[7] C. Achard, J. Devars, and L. lacassagne, "Object image retrieval with image compactness vectors.," 15th International Conference on Pattern Recognition, Bercelona, Spain, pp. 271-274, 2000.

[8] L. A. Zadeh, "Fuzzy sets.," Inform. and Control, vol. 8, pp. 338-353, 1965.

[9] S. K. Pal and A. Rosenfeld, "Image enhancement and thresholding by optimization of fuzzy compactness," in Fuzzy Models For pattern Recognition, James C. Bezdek and Sankar K. Pal, Eds., pp. 369-379. IEEE PRESS, 1991.

[10] A. Rosenfeld, "Fuzzy digital topology," in Fuzzy Models For pattern Recognition, James C. Bezdek and Sankar K. Pal, Eds., pp. 331-339. IEEE press, 1991.

[11] B. B. Chaudhury and B. Uma Shankar, "An efficient algorithm for extrema detection in digital images.," Pattern Recognition Letters, vol. 10, pp. 81-85, 1989.

[12] S. K. Pal and D. Dutta Majumder, Fuzzy mathematical Approach to Pattern Recognition, Willey Eastern Limited, New York, 1985.

[13] R. C. Gonzalez and R. E. Woods, Digital Image Processing, Wiley, New York, 1985.

[14] M. K. Kundu and S. K. Pal, "Thresholding for edge detection using human psychovisual phenomena," Pattern Recognition Letters, vol. 4, pp. 433-411, 1986.

[15] C. Achard, E. Bigorgne, and J. Devars, "A sub-pixel and multispectral corner detector," ICPR 2000, vol. 3, pp. 971-974, 2000.

[16] A. D. Bimbo, Visual Information Retrieval, Morgan Kaufmann Publishers,Inc., San francisco, USA, 2001. 